\title{
Squeezing of Light within the Framework of Population Theoretic Approach*
}

\author{
S. K. Srinivasan \\ Department of Mathematics, Indian Institute of Technology, Madras-600 036, India
}

Z. Naturforsch. 52a, 124-126 (1997)

A multiphase approach for the population model of photons is proposed and it is shown that number squeezed coherent light as well as thermal light can be accomodated. It is also shown that the multiphase approach is a very natural way of describing non-Markov nature of the evolution of the density matrix.

\section{Introduction}

The object of this contribution is to establish the viable nature of the population theoretic approach in modelling the properties of the output radiation from amplifiers. In earlier contributions [1, 2] we have shown that the population point process model of radiation in different contexts exhibits diverse properties of light such as bunching, leading to thermal behaviour, and anti-bunching. Using a multi-phase approach for the evolution of the population process, we have also shown [3] that the model of radiation corresponds to the amplitude mixture of coherent and chaotic light. In a more recent contribution [4] we have further demonstrated the possibility of accomodating squeezed coherent light at least to second order within the framework of population theory. In this contribution we establish the coherent nature of the resulting radiations to all orders besides proving that a sub-population of the assembly of photons is number squeezed in the sense that its variance is sub-Poissonian. Besides we also establish that the multi-phase approach is the most natural way of describing coarse-graining of the density matrix.

\section{Population Point Process Model}

We use as our starting point the Shepherd model [5] as adapted by Srinivasan [1]; the photon field is modelled as a discrete valued population, the field evolving in cavity as a birth (stimulated emission), death (ab-

* Presented at a Workshop in honor of E. C. G. Sudarshan's contributions to Theoretical Physics, held at the University of Texas in Austin, September 15-17, 1991.

Reprint requests to Prof. Dr. S. K. Srinivasan. sorption) and immigration (spontaneous emission) process. The field-detector interaction is modelled as an emigration process with a constant rate $\eta$ per individual. Likewise the death rate is assumed to be a constant $\mu$; however, the emission rate is not taken to be a constant and is specified as follows. The process of spontaneous emission, taken in isolation, is governed by a semi-Markov process $Z(t)$ over a finite set of elements $1,2, \ldots n$ with constant rates $\beta_{i}$ of transition, non-vanishing only for transitions of the type $i \rightarrow i+1(i=1,2, \ldots n-1)$ and $v_{i}$ for transitions of the type $n \rightarrow i(i=1,2, \ldots n)$; the spontaneous emission of a photon of index $j$ occurs whenever there is a transition of $Z(t)$ from $n \rightarrow i$, the process of spontaneous emission restarting from state $i$, the rate for which is taken to be $v_{j i}$ with $v_{i}=\Sigma_{j} v_{j i}$; we use the index $j$ to characterize the photon thus emitted. Likewise the process of stimulated emission due to any particular photon (of index $j$ ) is assumed to go through a semi-Markov process resulting in an emission. As emphasized earlier [1] the primary motivation to use a semi-Markov process proceeding through several states (with constant rates) is to handle the non-Markov evolution, which is otherwise difficult to deal with. The particular case when $n=1$ is straight forward [5] and leads to a thermal stream of photons. A very special cae of the sub class when $n=3$ was proposed [2] to describe a stream of anti-bunched photons. Yet another special case, when $n=3$, was studied [6] to describe a stream corresponding to the amplitude mixture of coherent and thermal streams.

To proceed further we introduce the following notation

$X_{i}(t)$ : the state of population of photons of index $j(i=l, 2, \ldots n)$. 
$X(t): \quad$ the total size of the population.

$Z(t): \quad$ the state process of spontaneous emissions taking values over the index set $1,2, \ldots n$. Members of the index set will be called phases, consistent with population theoretic notation.

$X_{i}^{j}(t): \quad$ the size of population of photons of index $i$ and phase $j .(i, j=1,2 \ldots n)$.

$g_{j}(w, t)$ : the conditional generating function of photons defined to be equal to

$$
E\left[w^{X_{i}(t)} \mid X(0)=X_{i}^{j}(0)=1, \beta_{k}=0: k=1,2, \ldots n\right]
$$$$
i, j=1,2, \ldots n \text {. }
$$

$G_{i}\left(w_{1}, w_{2}, \ldots, w_{n}, t\right):$ the generating function of photons of different indices denoted for brevity by $G_{i}(t)$, where

$$
\begin{array}{r}
G_{i}(t)=E\left[w_{1}^{X_{i}(t)} w^{X_{2}(t)} \ldots w_{n}^{X_{n}(t)} \mid X(O)\right. \\
=O, Z(O)=i] i=1,2, \ldots n,
\end{array}
$$

where $E$ stands for the mathematical expectation of the quantity within the brackets. The special conditioning introduced in the definition of $g_{i}(w, t)$ renders the contributing process immigration taboo and facilitates the solution (see [1], Chapter 4); it is also to be noted that by choice $g_{i}(w, t)$ is independent of $i$; the main motivation is one of simplicity in the first instance. Moreover it is also evident from earlier investigations that a non-trivial choice of evolution of $g_{j}$ does in general promote thermal behaviour. In this contribution, since we confine our attention to near coherent behaviour, we make the evolution of $g_{j}$ rather trivial:

$$
g_{j}\left(w_{i}, t\right)=\left(w_{i}-1\right) e^{-(\mu+\eta) t}+1 .
$$

By examining the process $\{\boldsymbol{Z}(t)\}$ and using the backward differential technique (see [1], Chapt. 4) we find

$$
\frac{\partial G_{i}(t)}{\partial t} i=\beta_{i} G_{i}(t)+\beta_{i} G_{i+1}(t)=1,2, \ldots n-1,
$$

$$
\frac{\partial G_{n}(t)}{\partial t}=-v_{n} G_{n}(t)+\sum_{i, j=1}^{n} v_{i, j} G_{j}(t) g_{1}\left(w_{i}, t\right)
$$

with the initial conditions

$$
G_{i}(0)=1, \quad i=1,2, \ldots n,
$$

where

$$
\beta_{n}=v_{1}+v_{2}+\ldots+v_{n}, v_{i}=\sum_{j} v_{i j} .
$$

At the outset we note that we need to characterize the steady state behaviour of the generating function. We show in particular that the steady state generating function for the total population corresponds to a coherent stream (Poisson distribution). The direct proof is rather difficult; we proceed as follows. First we look at the process $\{Z(t)\}$. Defining

$$
\pi_{i j}(t)=\operatorname{Pr}\{Z(t)=j \mid Z(O)=i\}
$$

we find

$\lim _{t \rightarrow \infty} \pi_{j i}(t)=\pi_{i}=\pi_{n}\left(J_{1}+J_{2}+\ldots+J_{i}\right) / L_{i}$,

$\frac{1}{\pi_{n}}=1+J_{1}+\frac{J_{1}+J_{2}}{L_{2}}+\ldots+\frac{J_{1}+J_{2}+\ldots J_{n-1}}{L_{n-1}}$,

where

$\beta_{i}=L_{i} \beta, v_{i}=J_{i} \beta ; i=1,2 \ldots n, L_{1}=1$.

Next we observe that our interest is in the function $G(w, t)$ where

$$
G(w, t)=\sum_{i} \pi_{i} G_{i}\left(w_{i}, w_{2}, \ldots w_{n}, t\right),
$$

where $w_{i}=w(i=1,2, \ldots n)$ on the r.h.s. Accordingly we multiply the system of equations (4) and (5) by appropriate constants to obtain

$$
\frac{\partial G(w, t)}{\partial t}=\pi_{n} \beta \sum J_{i} G_{i}(w-1) e^{-(\mu+\eta) t} .
$$

If we now impose the conditions

$$
J_{1}+J_{2}+\ldots+J_{i}=L_{i} J_{i} \quad i=2, \ldots n,
$$

then we find

$$
\frac{\partial G(w, t)}{\partial t}=-\beta G(w, t)(w-1) e^{-(\mu+\eta) t},
$$

which leads to

$$
G(w, t)=\exp -\left[\frac{\beta(w-1)\left(1-e^{-(\mu+\eta) t}\right.}{\mu+\eta}\right],
$$

proving that the steady state population of photons is Poisson in character. The set of conditions (14) in general will lead to a feasible choice of the rate constants $\beta_{i}$ and $v_{i}$.

We are now comfortably placed to draw the inferences from the above results. We introduce the moments by

$$
A_{i j}(t)=\frac{\partial G_{i}(t)}{\partial s_{j}}, \quad B_{i j k}(t)=\frac{\partial^{2} G_{i}(t)}{\partial w_{j} \sigma w_{k}},
$$


where the derivatives are evaluated at the point $w_{1}=w_{2}=\ldots w_{n}=1$. Setting $v_{1}=L \beta, v_{2}=M \beta$, we find that the constraint (14) leads to $M=1$. Setting $\mu+\eta=k \beta$, we obtain after some calculations the following results for the steady state population

$E\left[X_{1}(\infty)\right]=L /[K(L+1)], \quad E\left[X_{2}(\infty)\right]=1 /[K(L+1)]$, $E\left[X_{1}(\infty)\left\{X_{1}(\infty)-1\right\}\right]=B_{11}=L^{2} /\left[K^{2}(L+1)\right]$, $E\left[X_{2}(\infty)\left\{X_{2}(\infty)-1\right\}\right]$ $=B_{22}(K+1) /\left[K^{2}(L+1)(K+L+1)\right]$,

$B_{11}=B_{11} /\left[A_{1} 1(\infty)\right]^{2}=(L+1)(K+L+1)$,

$B_{22}=B_{11}(K+1)$.

Hence we infer that, while the total population is Poissonian, the stream corresponding to $X_{1}$ is squeezed in as much as $B_{11}<1$ and $B_{22}>1$ for all possible choices of $L$ and $K$.

An exactly similar situation [7] holds for the case $n=3$. Setting $v_{1}=L \beta, v_{2}=J B, v_{3}=M \beta, \beta_{2}=I \beta$, $\beta_{1}=\beta$, we find that the constraints (14) reduce to

$$
M=1, \quad I J=L+J,
$$

[1] S. K. Srinivasan, Point Process Modes of Cavity Radiation and Detection (Griffin, London 1988).

[2] S. K. Srinivasan, J. Phys. A Math. Gen. 22, 259 (1989).

[3] S. K. Srinivasan and V. Sridharan, J. Phys. A. Math. Gen. 23, 491 (1990).

[4] S. K. Srinivasan, J. Phys. A Math. Gen. 23, 369 (1990). and we find

$$
\begin{aligned}
B_{11} & =I(1+I J) / D, \quad B_{22}=B_{11}(K+1), \\
B_{33} & =B_{22}(1+K / I), \\
D & =K^{2}+K(I+1+I J)+I(1+I J),
\end{aligned}
$$

providing ample evidence for the existence of bimodal (number) squeezed streams.

The author is delighted to contribute this paper in the meeting organized to mark the sixtieth birthday of Professor E. C. G. Sudarshan who has contributed in a significant manner to the development of Particle Theory and Optics. While his early contribution in the form of $\mathrm{V}-\mathrm{A}$ theory has provided further impetus for the development of unified theory of interactions, his contribution to optics, and particularly the theory of over-complete states has formed the basis for further research in the past thirty years. While acknowledging the encouragement and support that the author has received from Professor Sudarshan in all his scientific endeavours, the author wishes him and his family a long, happy and prosperous life, besides many more decades of further fruitful research.

[5] M. Sargent III, M. Scully, and W. E. Lamb, Laser Physics, Addison Wesley, Reading 1974.

[6] T. Shepherd, Opt. Acta 28, 567 (1981).

[7] S. K. Srinivasan and V. Sridharan, J. Mod. Opt. 36, 711 (1989). 